\title{
On the Diversity of Network-Coded Cooperation With Decode-and-Forward Relay Selection
}

\author{
Thang X. Vu, Member, IEEE, Pierre Duhamel, Fellow, IEEE, and Marco Di Renzo, Senior Member, IEEE
}

\begin{abstract}
In this paper, we study outage probability (OP) and diversity order of a $M$-source and $N$-relay wireless network that combines network coding (NC) and relay selection (RS). More specifically, a decode-and-forward (DF) relaying protocol is considered and the network-encoding vectors at the relays are assumed to constitute a maximum distance separable (MDS) code. Single relay selection (SRS) and multiple relay selection (MRS) protocols are investigated, where the best relay and the $L$ best relays forward the network-coded packets to the destination, respectively. An accurate mathematical framework for computing the OP is provided and from its direct inspection the following conclusions on the achievable diversity are drawn: 1) the SRS protocol achieves diversity order equal to two regardless of $M$ and $N$ and 2) the MRS protocol achieves diversity order equal to $L+1$ if $L<M$ and equal to $N+1$ if $L \geq M$. These analytical findings are substantiated with the aid of Monte Carlo simulations, which also show that RS provides a better OP than NC based on repetition coding if $L \geq M$.
\end{abstract}

Index Terms-Network coding, cooperative networks, relay selection, outage probability, diversity order.

\section{INTRODUCTION}

$\mathbf{C}$ OOPERATIVE diversity is an effective technique for increasing the coverage and for improving the performance of wireless networks [1]. In cooperative networks, idle nodes, henceforth called relays, are allowed to overhear the packets transmitted from the sources and to forward their estimates to a destination. Since multiple copies of the same signal are received at the destination, a better end-to-end reliability is, in general, obtained. If multiple relays are available in the network and a Relay Selection (RS) protocol is used, in particular, full diversity order can be achieved [2].

In cooperative networks, in general, several sources may want to send data to the same destination with the aid of the same idle nodes. If each source uses the relays in a roundrobin fashion, the price to pay for achieving full diversity may be an unacceptable reduction of the spectral efficiency [3]. To

Manuscript received October 21, 2014; revised January 18, 2015; accepted March 23, 2015. Date of publication April 6, 2015; date of current version August 10, 2015. This work was supported in part by the European Commission through the FP7-NoE NEWCOM\# Project under Grant 318306. The associate editor coordinating the review of this paper and approving it for publication was L. Song.

T. X. Vu is with Singapore University of Technology and Design, Singapore 138682 (e-mail: xuanthang_vu@sutd.edu.sg).

P. Duhamel and M. Di Renzo are with Paris-Saclay University, Laboratory of Signals and Systems (UMR-8506), CNRS-CentraleSupelec-University ParisSud XI, Gif-sur-Yvette 91192, France (e-mail: pierre.duhamel@1ss.supelec.fr; marco.direnzo@1ss.supelec.fr).

Color versions of one or more of the figures in this paper are available online at http://ieeexplore.ieee.org.

Digital Object Identifier 10.1109/TWC.2015.2420098 overcome this limitation, Network-Coded Cooperation (NCC) has recently been introduced, where the relays are capable of retransmitting all the packets received from the sources in a single time-slot, by capitalizing on the concept of Network Coding (NC) [4]. Recently, several researchers have investigated the benefits of NC for application to relay-aided networks. In [5], the capacity region of NCC is derived and it is proved that NCC is capable of outperforming classical cooperative networks. In [6] and [7], the advantages of combining NC with Single Relay Selection (SRS) and Multiple Relay Selection (MRS) protocols for application to the Two-Way Relay Channel (TWRC) are investigated, respectively. In [7], a suboptimal RS criterion for application to the TWRC with Amplify-and-Forward (AF) is introduced and studied. A similar RS protocol is considered in [8], where Outage Probability (OP), Bit Error Rate (BER) and diversity order are studied. These papers, however, are focused on the analysis of bidirectional networks. The analysis of NCC with RS for application to unidirectional networks has, on the other hand, received less attention [9], [10].

Motivated by these considerations, we study the OP and the diversity order of NCC with RS in a multiple-access relayaided network that consists of $M$ sources, $N$ relays, and one destination. The considered system model may find application in the uplink of cellular networks, where a base station acts as the destination, some active mobile terminals act as the sources, and some idle mobile terminals act as the relays. This network setup has recently been investigated by other researchers. In [11], the Diversity Multiplexing Tradeoff (DMT) of NCC is studied and it is shown that full diversity can be achieved by using RS, under the assumption that the destination is able to successfully decode the packets of all the sources. In [12], the analysis in [11] is generalized by removing the abovementioned assumption and by studying SRS and MRS protocols. The correlation of the ordered statistics originating from the RS protocol is, however, not completely taken into account [7]. In [13]-[15], the authors study OP, BER, and diversity order of Analog Network Coding (ANC). In the present paper, on the other hand, we focus our attention on the analysis of OP and diversity order of Decode-and-Forward (DF-) based RS that uses Digital NC (DNC), where the network-encoding vectors at the relays are assumed to constitute a Maximum Distance Separable (MDS) code [10]. More specifically, the present paper provides the following contributions:

- Exact and asymptotic expressions of the OP for SRS and MRS protocols are derived, which highlight fundamental performance trends as a function of several system parameters. 


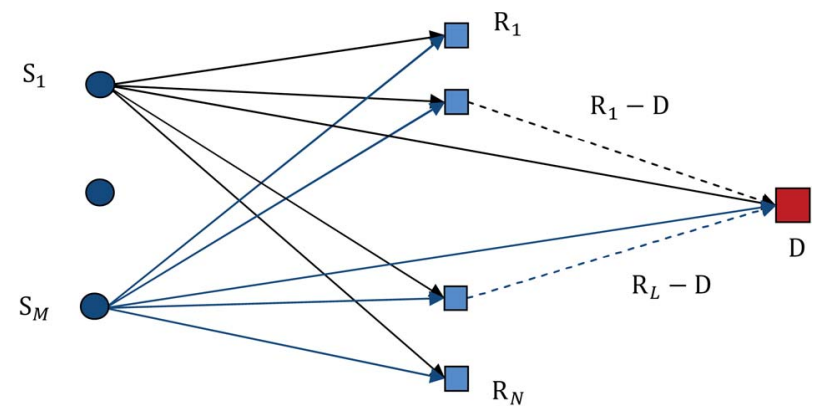

Fig. 1. $M$-source and $N$-relay wireless network combining NC and RS.

- From the asymptotic expression of the OP, the diversity order is obtained and the following conclusions are drawn: 1) the SRS protocol achieves a diversity order equal to two regardless of $M$ and $N$ and 2) the MRS protocol achieves a diversity order equal to $L+1$ if $L<$ $M$ and equal to $N+1$ if $L \geq M$.

Numerical results show that NCC with RS provides better performance than NCC with repetition-based transmission if $L \geq M$.

The remainder of the present paper is organized as follows. In Section II, the system model is introduced. In Section III, SRS and MRS protocols are described. In Section IV and Section V, the OP of SRS and MRS protocols is computed, respectively. In Section VI, the benefits of NCC with RS compared to NCC with repetition-based relaying are investigated. In Section VII, mathematical framework and findings are validated with the aid of Monte Carlo simulations. Finally, Section VIII concludes the present paper.

\section{SYSTEM MODEL}

We consider a cooperative network that consists of $M$ sources denoted by $S_{m}, 1 \leq m \leq M, N$ relays denoted by $R_{n}, 1 \leq n \leq N$, and one destination denoted by $D$, as depicted in Fig. 1. Perfect time synchronization is assumed and all channels experience block Rayleigh fading and Additive White Gaussian Noise (AWGN). All nodes are equipped with a single antenna and operate in a half-duplex mode. One cooperative period consists of two phases: broadcasting and relaying.

In the broadcasting phase, the sources transmit their data symbols to the relays and to the destination in non-overlapping time-slots. This phase, thus, lasts $M$ time-slots. Let $b_{m}$ denote the data symbol emitted by source $S_{m}, 1 \leq m \leq M$. Each $b_{m}$ symbol contains $q$ data bits. A $2^{q}$-order modulator maps $b_{m}$ into a signal symbol $x_{m}$. The signal received at relay $R_{n}, 1 \leq n \leq$ $N$, and at the destination can be formulated as follows:

$$
\left\{\begin{array}{l}
y_{S_{m} R_{n}}=\sqrt{P_{S_{m} R_{n}}} h_{S_{m} R_{n}} x_{m}+z_{S_{m} R_{n}} \\
y_{S_{m} D}=\sqrt{P_{S_{m} D}} h_{S_{m} D} x_{m}+z_{S_{m} D}
\end{array},\right.
$$

where $h_{X Y}$ for $X \in\left\{S_{m}\right\}$ and $Y \in\left\{R_{n}, D\right\}$ denotes the channel fading coefficient of the link $X \rightarrow Y$, which is a complex Gaussian random variable with zero mean and unit variance, i.e., $\mathbb{E}\left\{\left|h_{X Y}\right|^{2}\right\}=1$. All channels are assumed to be mutually independent; $P_{X Y}$ denotes the average received signal power at node $Y$ from node $X$, including the path loss; and $z_{(.)}$denotes the AWGN with zero mean and variance $\sigma^{2}$.

The relaying phase is based on the RS protocol and it is described in the next section. Let us assume that $L \leq M$ relays are selected for relaying the data of the sources to the destination. These selected relays execute the following operations. First, they decode the data received from the sources by using a Maximum Likelihood (ML) detector as follows:

$$
\hat{b}_{S_{m} R_{n}}=\arg \max _{\tilde{b}_{m}} \operatorname{Pr}\left\{\tilde{b}_{m} \mid y_{S_{m} R_{n}}\right\},
$$

where $\tilde{b}_{m}$ denotes the hypothesis of the data symbol transmitted by source $S_{m}$.

Then, they apply $\mathrm{NC}$ in $\mathrm{GF}\left(2^{q}\right)$ to the demodulated data and the network-coded symbol as follows is obtained:

$$
\hat{b}_{R_{n}}=\alpha_{S_{1} R_{n}} \hat{b}_{S_{1} R_{n}} \boxplus \ldots \boxplus \alpha_{S_{M} R_{n}} \hat{b}_{S_{M} R_{n}},
$$

where $\alpha_{(.)}$denotes the NC coefficients and $\boxplus$ denotes the sum operation in $\mathrm{GF}\left(2^{q}\right)$.

Finally, the network-coded symbol $\hat{b}_{R_{n}}$ is modulated into a signal symbol $\hat{x}_{R_{n}}$ and forwarded to the destination. Unlike [12], where only successfully decoded symbols are relayed, we assume that $\mathrm{NC}$ is applied to all, correct and incorrect, demodulated symbols. At the destination, this error propagation phenomenon can be mitigated by using appropriate demodulation schemes [10].

Let $\left\{i_{1}, \ldots, i_{L}\right\}$ denote the indices of the selected relays. The signal received at the destination from them can be formulated as follows $\left(n \in\left\{i_{1}, \ldots, i_{L}\right\}\right)$ :

$$
y_{R_{n} D}=\sqrt{P_{R_{n} D}} h_{R_{n} D} \hat{x}_{R_{n}}+z_{R_{n} D},
$$

where $h_{R_{n} D}$ represents the channel fading coefficient of the link $R_{n} \rightarrow D$, which is a complex Gaussian random variable with zero mean and unit variance; $P_{R_{n} D}$ denotes the received signal power at $D$ from $R_{n}$; and $z_{R_{n} D}$ denotes the complex AWGN with zero mean and variance $\sigma^{2}$.

\section{Relay Selection Protocols}

We assume that RS is implemented based on the suboptimal max-min selection criterion [6], which maximizes the worst end-to-end Signal-to-Noise-Ratio (SNR). In particular, two case studies are analyzed.

\section{A. Single Relay Selection}

Let us consider a generic source-to-relay-to-destination link. The end-to-end reliability of this dual-hop link is determined by the weakest link between the source-to-relay and the relayto-destination links. Likewise, let a generic multi-source-torelay link where NC is applied at the relays. The end-to-end reliability of this multi-point-to-point link is determined by the weakest source-to-relay link. As a result, each dual-hop multisource-to-relay-to-destination link turns out to be equivalent to a single-hop link with the SNR as follows [10]:

$$
\gamma_{n}=\min \left\{\gamma_{S_{1} R_{n}}, \ldots, \gamma_{S_{M} R_{n}}, \gamma_{R_{n} D}\right\}
$$

where $\gamma_{X Y} \triangleq \frac{P_{X Y}\left|h_{X Y}\right|^{2}}{\sigma^{2}}$ denotes the instantaneous SNR of the link $\mathrm{X} \rightarrow \mathrm{Y}$. 
Since the SNRs $\gamma_{X Y}$ in (5) are mutually independent and exponentially distributed (Rayleigh fading is assumed), the $\mathrm{Cu}$ mulative Distribution Function (CDF) of $\gamma_{n}$ can be formulated as follows [16]:

$$
F_{\gamma_{n}}(x)=1-e^{-\frac{x}{\bar{\gamma}_{n}}}
$$

where

$$
\frac{1}{\bar{\gamma}_{n}}=\frac{1}{\bar{\gamma}_{S_{1} R_{n}}}+\ldots+\frac{1}{\bar{\gamma}_{S_{M} R_{n}}}+\frac{1}{\bar{\gamma}_{R_{n} D}}
$$

and $\bar{\gamma}_{X Y}=\frac{P_{X Y}}{\sigma^{2}}$ denotes the average SNR of the link $X \rightarrow Y$.

In SRS, the relay with the highest equivalent SNR $\gamma_{n}$ in (5) is selected for forwarding the data of the sources, since this maximizes the end-to-end performance. The SNR of the selected relay, denoted by $g_{S R S}$, can be formulated as follows:

$$
g_{S R S}=\max \left\{\gamma_{1}, \ldots, \gamma_{N}\right\} .
$$

With the aid of [16], the CDF of $g_{S R S}$, denoted by $F_{g_{S R S}}(\cdot)$, can be formulated as follows:

$$
F_{g_{S R S}}(x)=\prod_{n=1}^{N} F_{\gamma_{n}}(x)=\prod_{n=1}^{N}\left(1-e^{-\frac{x}{\bar{\gamma}_{n}}}\right) .
$$

After the relay selection process, the selected relay forwards the network-coded symbols to the destination.

\section{B. Multiple Relay Selection}

The end-to-end reliability of SRS can be improved by allowing more than one relay to forward data of the sources to the destination. In the MRS protocol, the $1<L \leq M$ relays with the highest SNRs in (5) are selected for data forwarding. In practice, the MRS protocol can be implemented in a distributed fashion as proposed in [2]. It is worth mentioning that the RS protocol considered in this paper is different from that used in [12], where a relay might be chosen several times.

With the aid of the same notation as for the SRS protocol, the SNRs of the $L$ best relays can be formulated as $\mathbf{g}_{M R S}=$ $\left\{g_{1}, \ldots, g_{L}\right\}$, where $g_{l}=\max ^{l}\left\{\gamma_{1}, \ldots, \gamma_{N}\right\}$ denotes the $l^{t h}$ maximum of the set $\left\{\gamma_{1}, \ldots, \gamma_{N}\right\}$, which can be formally defined as follows:

$$
\begin{aligned}
\operatorname{Pr}\left\{g_{l} \leqslant x\right\}=\operatorname{Pr}\left\{\left(N-l+1 \text { values } \gamma_{n} \leqslant x\right)\right. \\
\left.\cap\left(l-1 \text { values } \gamma_{n}>x\right)\right\} .
\end{aligned}
$$

The OP of the MRS protocol depends on the distribution of $\mathbf{g}_{M R S}$, which in turn depends on the distribution of $g_{l}$ for $l=$ $1,2, \ldots, L$. The following two lemmas provide the distribution of $g_{l}$, which is used in Section V for studying the end-to-end outage of MRS.

Lemma 1: The CDF, $F_{g_{l}}(\cdot)$, of $g_{l}=\max ^{l}\left\{\gamma_{1}, \ldots, \gamma_{N}\right\}$ for $1 \leq l \leq L$, where $\gamma_{n}$ for $1 \leq l \leq N$ is defined in (5) can be formulated as follows:

$$
F_{g_{l}}(x)=\sum_{k=1}^{l}(-1)^{k-1} \mathcal{C}_{N-l+k}^{N-l+1} \Gamma_{l}(k, x),
$$

where $\mathcal{C}_{k}^{n}=\frac{n !}{(n-k) ! \times k !}$ denotes the binomial coefficient and

$$
\Gamma_{l}(k, x)=\sum_{\substack{i_{1}=1, \ldots, i_{N-l+k}=1 \\ i_{1} \neq \ldots \neq i_{N-l+k}}}^{N} \prod_{n=i_{1}}^{i_{N-l+k}} F_{\gamma_{n}}(x),
$$

as well as, for notational simplicity, the following shorthand is introduced:

$\sum_{\substack{i_{1}=1, \ldots, i_{k}=1 \\ i_{1} \neq \ldots \neq i_{k}}}^{n}\left(\prod_{j=i_{1}}^{i_{k}} x_{j}\right)=\sum_{i_{1}=1}^{n-k+1} \ldots \sum_{i_{k}=i_{k-1}+1}^{n}\left(x_{i_{1}} \ldots x_{i_{k}}\right)$

Proof: Let $i_{1}, \ldots, i_{N-l+1}, \ldots, i_{N}$ denote the indices that satisfy (10), i.e., $\gamma_{n}<x$ for $i_{1} \leq n \leq i_{N-l+1}$ and $\gamma_{n}>x$ for $i_{N-l+1}<n \leq i_{N}$. Since the SNRs $\gamma_{n}$ are mutually independent, the probability in (10) is $\prod_{n=i_{1}}^{i_{N-l+1}} F_{\gamma_{n}}(x) \times$ $\prod_{n^{\prime}=i_{N-l+1}}^{i_{N}}\left(1-F_{\gamma_{n^{\prime}}}(x)\right)$ and there are $\mathcal{C}_{l-1}^{N}$ combinations of indices in (10) that possibly satisfy the condition. By applying polynomial factorization of the probability of each set of indices and computing the summation, the proof follows.

Lemma 2: Let the SNRs be independent and identically distributed (i.i.d.), i.e., $\bar{\gamma}_{1}=\ldots=\bar{\gamma}_{N}=\bar{\gamma}$. The diversity order of $g_{l}$ is $N-l+1$.

Proof: From (11), it follows that the asymptotic behavior of the CDF of $g_{l}$ depends on the terms $\Gamma_{l}(k, x)$, for $1 \leq k \leq$ $l$, that decay fastest with the SNR. If the channels are i.i.d., we have $\Gamma_{l}(1, x)=\mathcal{C}_{N-l+1}^{N}\left(F_{\gamma_{n}}(x)\right)^{N-l+1}$. For high-SNR, we have $F_{\gamma_{n}}(x) \rightarrow \frac{1}{\bar{\gamma}}$, which leads to $\Gamma_{l}(1, x) \rightarrow \mathcal{C}_{N-l+1}^{N} \frac{1}{\bar{\gamma}^{N-l+1}}$. This concludes the proof.

After MRS process, the $L$ selected relays forward the network-coded symbols to the destination.

\section{OUtage Probability of SRS}

\section{A. Exact Outage Probability of SRS}

The mutual information of a generic single-hop link with instantaneous SNR equal to $\gamma$ can be formulated as follows:

$$
I(\gamma)=\log _{2}(1+\gamma) .
$$

As a result, the per-link OP can be formulated as follows:

$$
P_{\text {out }}=\operatorname{Pr}\{I<R\}=\operatorname{Pr}\{\gamma<\underbrace{2^{R}-1}_{\gamma_{\text {th }}}\},
$$

where $\gamma_{t h}$ denotes the reliability threshold corresponding to the rate $R$.

As for the SRS protocol, the destination receives $M+1$ packets: $M$ from the sources and 1 from the best relay. An outage occurs if the destination is not able to decode the packets of all the sources, i.e., fewer than $M$ packets are correctly decoded. In other words, the system is not in outage if there is no more than one link whose SNR is below the threshold $\gamma_{t h}$. Accordingly, the OP of SRS can be formulated as 
$\mathrm{OP}_{S R S}=1-\overline{\mathrm{OP}}$, where $\overline{\mathrm{OP}}$ is the probability that the system is not in outage, which can be written as follows:

$$
\overline{\mathrm{OP}}=\operatorname{Pr}\left\{0 \text { links }<\gamma_{t h}\right\}+\operatorname{Pr}\left\{1 \text { link }<\gamma_{t h}\right\} .
$$

Theorem 1: Consider a cooperative network consisting of $M$ sources, $N$ relays, and one destination. Let $\mathrm{NC}$ be applied at the relays. The exact OP of SRS is as follows:

$$
\begin{gathered}
\mathrm{OP}_{S R S}=1-\prod_{m=1}^{M}\left(1-F_{\gamma_{S_{m} D}}\left(\gamma_{t h}\right)\right)+\left(1-F_{g_{S R S}}\left(\gamma_{t h}\right)\right) \\
\times \sum_{m=1}^{M}\left(F_{\gamma_{S_{m} D}}\left(\gamma_{t h}\right) \prod_{m \neq m^{\prime}=1}^{M}\left(1-F_{\gamma_{S_{m^{\prime}}}}\left(\gamma_{t h}\right)\right)\right),
\end{gathered}
$$

where $F_{\gamma_{S_{m} D}}(x)=1-e^{-\frac{x}{\bar{\gamma}_{S_{m} D}}}, F_{\gamma_{S_{m^{\prime}} D}}(x)=1-e^{-\frac{x}{\bar{\gamma}_{S_{m^{\prime}} D}}}$, and $F_{g_{S R S}}(\cdot)$ is given in (9).

Proof: Since the $M+1$ channels are mutually independent, we have $\operatorname{Pr}\left\{\gamma_{S_{1} D} \leq \gamma_{t h}, \gamma_{S R S} \leq \gamma_{t h}\right\}=\operatorname{Pr}\left\{\gamma_{S_{1} D} \leq\right.$ $\left.\gamma_{t h}\right\} \times \operatorname{Pr}\left\{\gamma_{S R S} \leq \gamma_{t h}\right\}$. The proof follows from (9) with the aid of some algebraic manipulations.

Corollary 1: Consider the same assumption as in Theorem 1. If the channels are i.i.d., i.e., $\bar{\gamma}_{S_{m} R_{n}}=\bar{\gamma}_{S_{m} D}=\bar{\gamma}_{R_{n} D}=$ $\bar{\gamma}, \forall n, m$, the OP of SRS can be formulated as follows:

$$
\begin{aligned}
\mathrm{OP}_{S R S}^{i i d}=1-e^{-\frac{M \gamma_{t h}}{\bar{\gamma}}} & +M e^{-\frac{(M-1) \gamma_{t h}}{\bar{\gamma}}}\left(1-e^{-\frac{\gamma_{t h}}{\bar{\gamma}}}\right) \\
\times & {\left[1-\left(1-e^{-\frac{(M+1) \gamma_{t h}}{\bar{\gamma}}}\right)^{N}\right] . }
\end{aligned}
$$

Proof: It follows directly from (17).

\section{B. Asymptotic Outage Probability of SRS}

The OP of SRS in Theorem 1 is a complex function of all average SNRs in the network, which does not allow one to get insightful information about the achievable diversity order. Theorem 2 provides a simpler asymptotic expression of the OP, which yields direct information on the diversity order.

Theorem 2: Consider a cooperative network consisting of $M$ sources, $N$ relays, and one destination. Let NC be applied at the relays. In the high-SNR regime, the asymptotic OP of SRS can be formulated as follows:

$$
\mathrm{OP}_{S R S}^{A s y m}=\sum_{\substack{m=1, m^{\prime}=1 \\ m \neq m^{\prime}}}^{M} \frac{\gamma_{t h}^{2}}{\bar{\gamma}_{S_{m} D} \bar{\gamma}_{S_{m^{\prime}} D}} .
$$

Proof: See Appendix A.

Theorem 2 highlights that the SRS protocol is capable of achieving only a diversity order equal to two regardless of the number of available relays. In particular, the asymptotic OP depends only on the source-to-destination links and it is independent of the source-to-relay and relay-to-destination links. These performance trends are different compared to their counterparts in single-source relay networks where $\mathrm{NC}$ is not used [2], as well as in TWRC with NC [6]. In these latter cases, in fact, full diversity order equal to $N$ is achieved.

\section{Outage Probability of MRS}

In this section, we analyze the OP of the MRS protocol. We assume that the network encoding vectors employed by the $L$ best selected relays constitute a MDS code, i.e., all encoding vectors are mutually independent [17]. As a result, the destination is capable of recovering the data of the sources after receiving at least $M$ error-free packets, either from the sources or from the active relays.

\section{A. Exact Outage Probability of MRS}

In the MRS protocol, the destination receives $M+L$ packets: $M$ from the sources and $L$ from the best relays. An outage occurs if the destination is not able to decode the packets of all the sources, i.e., fewer than $M$ packets are correctly decoded. In other words, the system is in outage if fewer than $M$ SNRs at the destination are above the reliability threshold $\gamma_{t h}$. In formulas, the $\mathrm{OP}$ can be written as follows:

$$
\begin{aligned}
\mathrm{OP}=\operatorname{Pr}\{0 \text { links }> & \left.\gamma_{t h}\right\}+\operatorname{Pr}\left\{1 \text { link }>\gamma_{t h}\right\} \\
& +\ldots+\operatorname{Pr}\left\{M-1 \text { links }>\gamma_{t h}\right\} .
\end{aligned}
$$

Theorem 3: Consider a cooperative network consisting of $M$ sources, $N$ relays, and one destination. Let NC be applied at the relays and the encoding vectors constitute a MDS code. If $L<$ $M$, the OP of the MRS protocol can be formulated as follows:

$$
\begin{array}{r}
\mathrm{OP}_{M R S}^{(1)}=\left(\sum_{m=0}^{M-L-1} \Psi(m)\right)\left(1+\sum_{l=1}^{L-1} F_{g_{l}}\left(\gamma_{t h}\right)\right) \\
+\sum_{k=1}^{L}\left(\Psi(M-k) \sum_{l=1}^{k} F_{g_{l}}\left(\gamma_{t h}\right)\right),
\end{array}
$$

otherwise the OP of MRS can be formulated as follows:

$$
\mathrm{OP}_{M R S}^{(2)}=\sum_{k=1}^{M}\left(\Psi(M-k) \sum_{l=1}^{k} F_{g_{l}}\left(\gamma_{t h}\right)\right),
$$

where $F_{g_{l}}(\cdot)$ is given in Lemma 1 and:

$$
\begin{aligned}
& \Psi(k)=\sum_{\substack{i_{1}=1, \ldots, i_{k}=1 \\
i_{1} \neq \ldots \neq i_{k}}}^{M}\left\{\prod_{m=i_{1}}^{i_{k}}\left(1-F_{\gamma_{S_{m} D}}\left(\gamma_{t h}\right)\right)\right. \\
&\left.\times \prod_{\substack{m^{\prime}=1 \\
m^{\prime} \neq\left\{i_{1}, \ldots, i_{k}\right\}}}^{M} F_{\gamma_{S_{m^{\prime}}}}\left(\gamma_{t h}\right)\right\} .
\end{aligned}
$$

Proof: See Appendix B.

Similar to the SRS protocol, the exact OP of the MRS protocol is a complex function of all the SNRs in the network, which does not allow an easy checking of the system diversity order. 


\section{B. Asymptotic Outage Probability of MRS}

Theorem 4: Consider a cooperative network consisting of $M$ sources, $N$ relays, and one destination. Let NC be applied at the relays and the encoding vectors constitute a MDS code. In the high-SNR regime, the asymptotic OP of the MRS protocol can be formulated as follows:

$$
\mathrm{OP}_{M R S}^{(1-\text { Asym })}=\sum_{\substack{i_{1}=1, \ldots, i_{L+1}=1 \\ i_{1} \neq \ldots \neq i_{L+1}}}^{M}\left(\prod_{m=i_{1}}^{i_{L+1}} \frac{\gamma_{t h}}{\bar{\gamma}_{S_{m} D}}\right)
$$

if $L<M$; otherwise, the $\mathrm{OP}$ is as follows:

$$
\mathrm{OP}_{M R S}^{(2-\text { Asym })}=\sum_{k=1}^{M} \Omega_{S}(k) \Omega_{R}(N-k+1),
$$

where:

$$
\Omega_{S}(k)=\sum_{\substack{i_{1}=1, \ldots, i_{k}=1 \\ i_{1} \neq \ldots \neq i_{k}}}^{M}\left(\prod_{m=i_{1}}^{i_{k}} \frac{\gamma_{t h}}{\bar{\gamma}_{S_{m} D}}\right)
$$

and:

$$
\Omega_{R}(k)=\sum_{\substack{i_{1}=1, \ldots, i_{k}=1 \\ i_{1} \neq \ldots \neq i_{k}}}^{N}\left(\prod_{n=i_{1}}^{i_{k}} \frac{\gamma_{t h}}{\bar{\gamma}_{n}}\right)
$$

Proof: From Lemma 2, we know that $F_{g_{l}}(\cdot)$ has diversity order equal to $N-l+1$. From (23), we note that $\Psi(\cdot)$ has diversity order equal to $M-k$. If $L<M$, the OP of MRS is given in (21). For high-SNR values, the first addend of (21), i.e., $\left(\sum_{m=0}^{M-L-1} \Psi(m)\right)\left(1+\sum_{l=1}^{L-1} F_{g_{l}}\left(\gamma_{t h}\right)\right)$, is dominated by $\Psi(M-L-1)$. By using the approximation in (40) and by keeping the dominant elements, we obtain (24). If $L \geq M$, the OP of MRS is given in (22). For every $k, \Psi(M-k)$ and $\sum_{l=1}^{k} F_{g_{l}}\left(\gamma_{t h}\right)$ have a diversity order equal to $k$ and to $N-k+1$, respectively. Therefore, $\Omega_{S}(k) \Omega_{R}(N-k+1)$ has diversity order equal to $N+1$ for every $k$. By still applying the approximation in (40), we obtain (25).

Theorem 4 shows that the MRS protocol is capable of achieving a diversity order equal to $L+1$ if $L<M$ and full diversity order equal to $N+1$ if $L \geq M$. It is worth noting that the diversity order for $L \geq M$ is different from that obtained in [12, Theorem. 3]. The reason is that the correlation originating from the ordered statistics of the SNRs of the selected relays is ignored in [12].

Theorem 4, in addition, highlights that the asymptotic OP of the MRS protocol does not depend on the relay channels if $L<$ $M$. This implies that the OP is independent of the number of available relays $N$ and of their spatial locations. The relays may, for example, be randomly deployed without affecting the OP [18]. If $L \geq M$, on the other hand, the asymptotic OP depends on $N$, on the network topology, and on the spatial locations of the relays. In this case, selecting more relays does not improve the system performance.

If $L=1$, Theorem 3 and Theorem 4 reduce, as expected, to Theorem 1 and to Theorem 2, respectively.

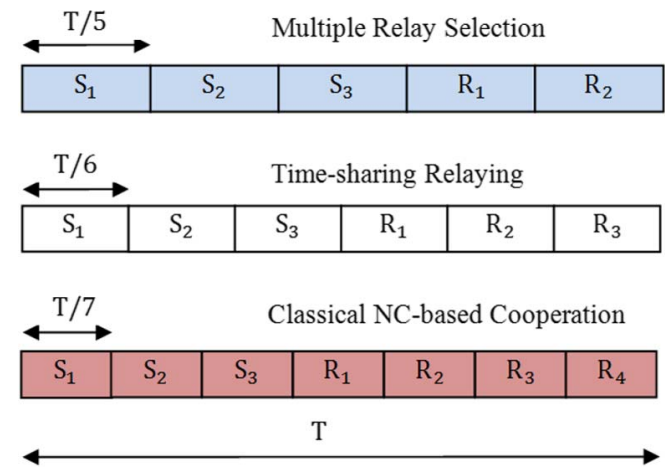

Fig. 2. Example of MRS-based NCC, time-sharing relaying and refectionbased NCC. Setup: $M=3, N=4$ and $L=2$.

\section{COMPARISON With STATE-OF-ThE-ART RElaying}

In this section, we compare MRS-based NCC against stateof-the-art relaying protocols, which include time-sharing relaying and NCC based on repetition coding. In particular, time-sharing relaying does not use NC and repetition-based NCC relies on all the available relays without using RS. For a fair comparison, the protocols are compared by assuming the same spectral efficiency. As example is illustrated in Fig. 2.

More specifically, let $T$ be the total transmission time, including broadcasting and relaying. It must be the same for all protocols, to guarantee the same spectral efficiency. As a consequence, the time-slot duration is different for NCC based on MRS, time-sharing relaying, and NCC based on repetition coding. As for NCC based on MRS, the duration of a time slot is $\tau_{M R S}=T /(M+L)$. Let $\mathcal{R}$ denote the system rate in bit per channel use. Thus, the rate in (15) for the MRS-based NCC, $\mathcal{R}_{M R S}$, is equal to

$$
\mathcal{R}_{M R S}=\frac{\mathcal{R}}{M+L} .
$$

Further details about time-sharing relaying and NCC based on repetition coding are provided in what follows.

\section{A. Time-Sharing Relaying}

In time-sharing relaying, each source relies on the help of the best available relay but NC is not applied. Consequently, the total time for completing the transmission from $M$ sources is $2 M$ time-slots.

In particular for the source $S_{m}$, the SNR of the best relay is chosen as follows [2]:

$$
g_{m}^{T S}=\max _{n=1, \ldots, N}\left\{\gamma_{n}^{T S}\right\},
$$

where:

$$
\gamma_{n}^{T S}=\min \left\{\gamma_{S_{m} R_{n}}, \gamma_{R_{n} D}\right\} .
$$

This implies that different sources may select different relays. The generic source $S_{m}$ is in outage if both $\gamma_{S_{m} D}$ and $g_{m}^{T S}$ are below the reliability threshold $\gamma_{t h}^{T S}$. In formulas:

$$
\begin{aligned}
\mathrm{OP}_{m} & =\operatorname{Pr}\left\{\gamma_{S_{m} D} \leq \gamma_{t h}^{T S}, g_{m}^{T S} \leq \gamma_{t h}^{T S}\right\} \\
& =\left(1-e^{-\frac{\gamma_{t h}^{T S}}{\bar{\gamma}_{S_{m} D}}}\right) \prod_{n=1}^{N}\left(1-e^{-\frac{\gamma_{t h}^{T S}}{\bar{\gamma}_{n}^{T S}}}\right),
\end{aligned}
$$


where

$$
\frac{1}{\bar{\gamma}_{t h}^{T S}}=\frac{1}{\bar{\gamma}_{S_{m} R_{n}}}+\frac{1}{\bar{\gamma}_{R_{n} D}} .
$$

In a multi-source network, by definition, an outage occurs if at least one source is in outage. Let $\overline{\mathrm{OP}}_{m}=1-\mathrm{OP}_{m}$ be the probability that source $S_{m}$ is not in outage. The OP of timesharing relaying can be formulated as follows:

$$
\mathrm{OP}_{T S}=1-\prod_{m=1}^{M} \overline{\mathrm{OP}}_{m}=1-\prod_{m=1}^{M}\left(1-\mathrm{OP}_{m}\right),
$$

where $\mathrm{OP}_{m}$ is given in (30).

By using the same line of thought as Theorem 2, the asymptotic (in the high-SNR regime) OP can be formulated as follows:

$$
\mathrm{OP}_{T S}^{\text {Asym }}=\sum_{m=1}^{M}\left(\frac{\left(\gamma_{t h}^{T S}\right)^{N+1}}{\bar{\gamma}_{S_{m} D}} \prod_{n=1}^{N}\left(\frac{1}{\bar{\gamma}_{S_{m} R_{n}}}+\frac{1}{\bar{\gamma}_{R_{n} D}}\right)\right) .
$$

From (32), it follows that time-sharing relaying achieves full diversity order equal to $N+1$. Achieving full diversity order, however, does not guarantee that time-sharing relaying outperforms MRS-based NCC, since, by assuming the same spectral efficiency, the reliability thresholds of the two protocols are, in general, different, i.e., $\gamma_{t h}^{T S} \neq \gamma_{t h}$. Let, in fact, the time-slot duration of time-sharing relaying be equal to $\tau_{T S}=T /(2 M)$. Then, the corresponding rate is equal to $\mathcal{R}_{T S}=\mathcal{R} /(2 M)$. By re-writing it in terms of $\mathcal{R}_{M R S}=\mathcal{R} /(M+L)$, we have $\mathcal{R}_{T S}=((2 M) /(M+L)) \mathcal{R}_{M R S}$. As a result, $\gamma_{t h}^{T S}=2^{\mathcal{R}_{T S}}-$ $1 \neq \gamma_{t h}=2^{\mathcal{R}_{M R S}}-1$.

\section{B. Repetition-Based (or Classical) NCC}

In repetition-based NCC, all the available relays forward network-coded symbols to the destination without using RS. The destination receives $M+N$ packets at the end of broadcasting and relaying phases. Hence, the total time for completing the transmission from the $M$ sources is $M+N$ time-slots.

In the light of the MDS assumption for the encoding vectors used at the relays, the destination is capable of retrieving the data of the $M$ sources if it receives at least $M$ correct packets out of the $M+N$ received ones. Let $\boldsymbol{g}^{C N C}=$ $\left\{\gamma_{S_{1} D}, \ldots, \gamma_{S_{M} D}, \gamma_{1}, \ldots, \gamma_{N}\right\}$ denote the vector of all the SNRs, where $\gamma_{n}=\min \left\{\gamma_{S_{1} R_{n}}, \ldots, \gamma_{S_{M} R_{n}}, \gamma_{R_{n} D}\right\}$ for $n=$ $1,2, \ldots, N$. The OP of repetition-based NCC can be formulated as follows:

$$
\begin{aligned}
\mathrm{OP}_{C N C} & =\operatorname{Pr}\left\{0 \text { links in } \boldsymbol{g}^{C N C}>\gamma_{t h}^{C N C}\right\} \\
& +\ldots+\operatorname{Pr}\left\{M-1 \text { links in } \boldsymbol{g}^{C N C}>\gamma_{t h}^{C N C}\right\}
\end{aligned}
$$

where $\gamma_{t h}^{C N C}$ denotes the reliability threshold.

By using a line of thought similar to NCC based on MRS, the OP can be formulated as follows:

$$
\mathrm{OP}_{C N C}=\sum_{k=1}^{M-1} \Psi_{C N C}(k),
$$

where

$$
\begin{array}{r}
\Psi_{C N C}(k)=\sum_{\substack{i_{1}=1, \ldots, i_{k}=1 \\
i_{1} \neq \ldots \neq i_{k}}}^{M+N}\left[\prod_{n=i_{1}}^{i_{k}}\left(1-F_{g_{n}^{C N C}}\left(\gamma_{t h}^{C N C}\right)\right)\right. \\
\left.\times \prod_{1=n^{\prime} \neq\left\{i_{1}, \ldots, i_{k}\right\}}^{M+N} F_{g_{n^{\prime}}^{C N C}}\left(\gamma_{t h}^{C N C}\right)\right] .
\end{array}
$$

Likewise, the asymptotic OP is as follows:

$\mathrm{OP}_{C N C}^{1-A s y m p}=\Omega_{S}(N+1)+\sum_{k=1}^{N} \Omega_{S}(k) \Omega_{C N C}(N-k+1)$,

if $N<M$; otherwise it is equal to:

$$
\mathrm{OP}_{C N C}^{2-A s y m p}=\sum_{k=1}^{M} \Omega_{S}(k) \Omega_{C N C}(N-k+1),
$$

where

$$
\Omega_{S}(k)=\sum_{\substack{i_{1}=1, \ldots, i_{k}=1 \\ i_{1} \neq \ldots \neq i_{k}}}^{M}\left(\prod_{m=i_{1}}^{i_{k}} \frac{\gamma_{t h}^{C N C}}{\bar{\gamma}_{S_{m} D}}\right)
$$

and

$$
\Omega_{C N C}(k)=\sum_{\substack{i_{1}=1, \ldots, i_{k}=1 \\ i_{1} \neq \ldots \neq i_{k}}}^{N}\left(\prod_{n=i_{1}}^{i_{k}} \frac{\gamma_{t h}^{C N C}}{\bar{\gamma}_{n}}\right)
$$

From (36) and (37), we observe that repetition-based NCC achieves full diversity order equal to $N+1$. Similar to the timesharing case, this does not necessarily imply that repetitionbased NCC outperforms MRS-based NCC. The respective reliability thresholds, in fact, are, in general, different, i.e., $\gamma_{t h}^{C N C} \neq \gamma_{t h}$. Let, in fact, the time-slot duration of repetitionbased NCC be equal to $\tau_{C N C}=T /(M+N)$. Then, the corresponding rate is equal to $\mathcal{R}_{C N C}=\mathcal{R} /(M+N)$. By re-writing it in terms of $\mathcal{R}_{M R S}=\mathcal{R} /(M+L)$, we have $\mathcal{R}_{C N C}=$ $((M+N) /(M+L)) \mathcal{R}_{M R S} \geq \mathcal{R}_{M R S}$. As a result, $\gamma_{t h}^{C N C}=$ $2^{\mathcal{R}_{C N C}}-1 \geq \gamma_{t h}=2^{\mathcal{R}_{M R S}}-1$.

When the number of the selected relays exceeds the number of the sources, i.e., $L \geq M$, by comparing (25) and (37) we conclude that MRS-based NCC always outperforms repetitionbased NCC because $\gamma_{t h}^{C N C} \geq \gamma_{t h}^{M R S}$.

\section{NUMERICAL RESULTS}

In this section, selected numerical results are illustrated to substantiate our findings. For ease of illustration, a symmetric network topology is considered, where $\bar{\gamma}_{S_{m} D}=\bar{\gamma}_{S D}, \forall m$, $\bar{\gamma}_{R_{n} D}=\bar{\gamma}_{R D}, \forall n$, and $\bar{\gamma}_{S_{m} R_{n}}=\bar{\gamma}_{S R}, \forall m, n$. For a given rate $\mathcal{R}$, the reliability thresholds are computed as discussed in Section VI. 


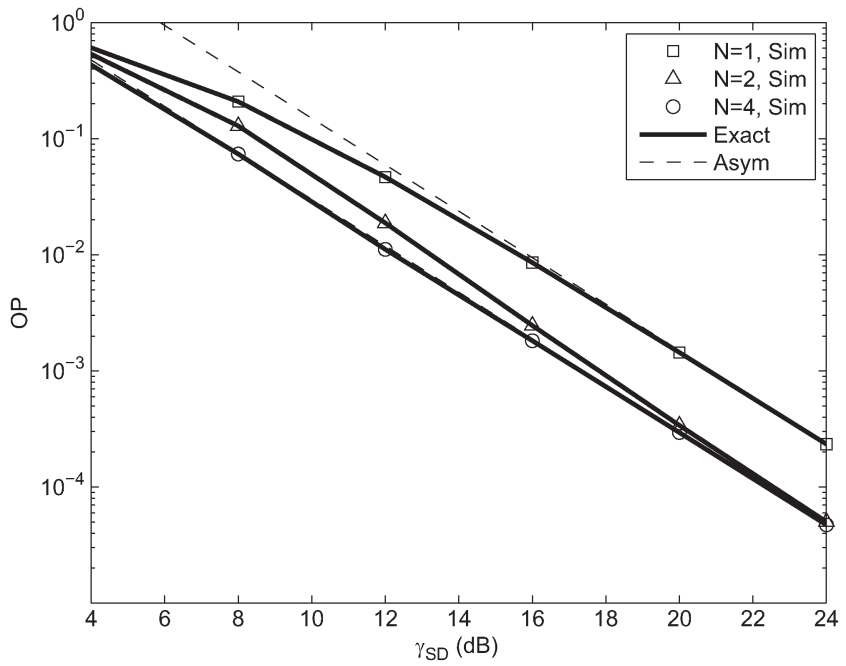

(a)

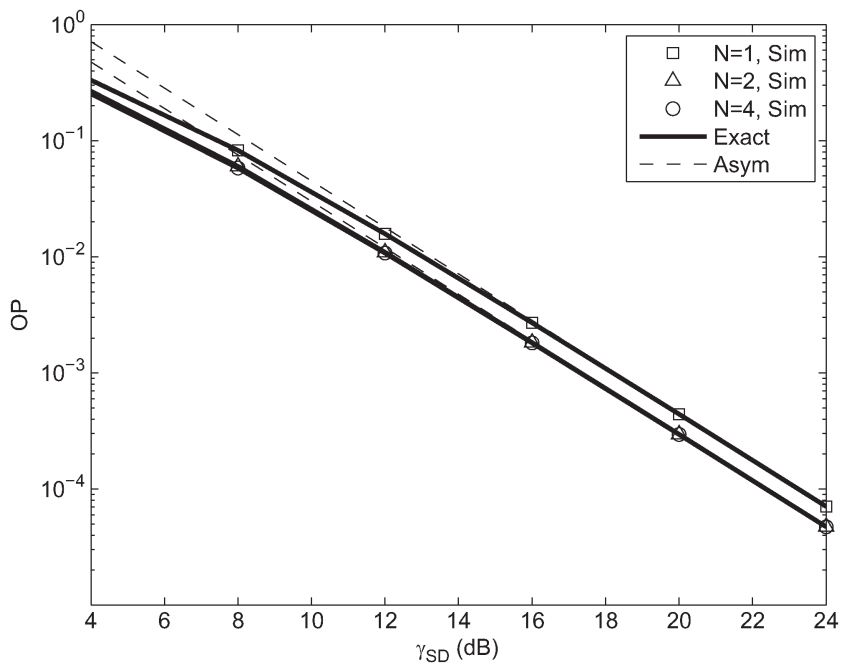

(b)

Fig. 3. OP of SRS as a function of $N$. Setup: $M=3$ and $\mathcal{R}=3 / 4$. (a) $\bar{\gamma}_{S D}=\bar{\gamma}_{S R}=\bar{\gamma}_{R D}$. (b) $\left(\bar{\gamma}_{S D}, \bar{\gamma}_{S R}, \bar{\gamma}_{R D}\right)=(\bar{\gamma}, \bar{\gamma}+9 \mathrm{~dB}, \bar{\gamma}+9 \mathrm{~dB})$.

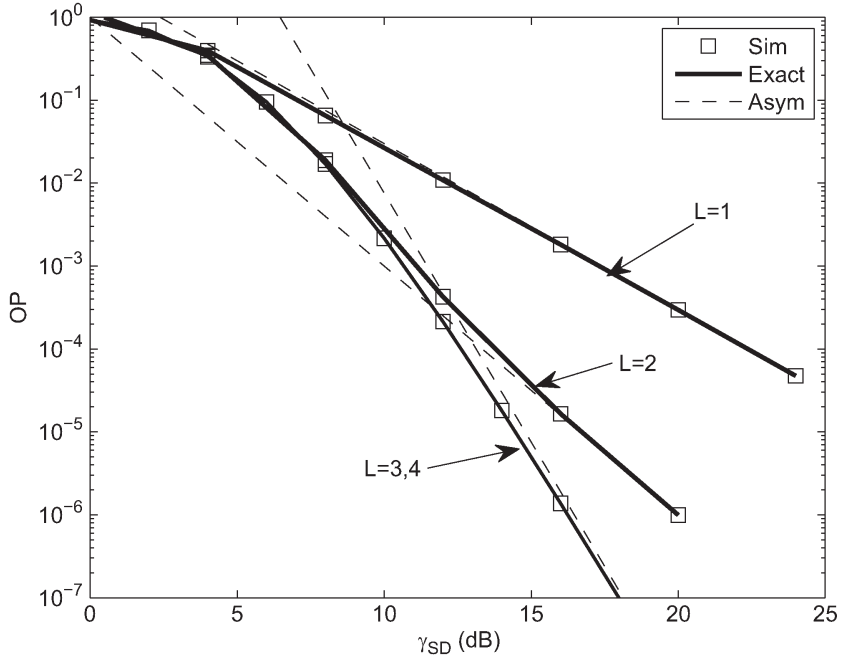

(a)

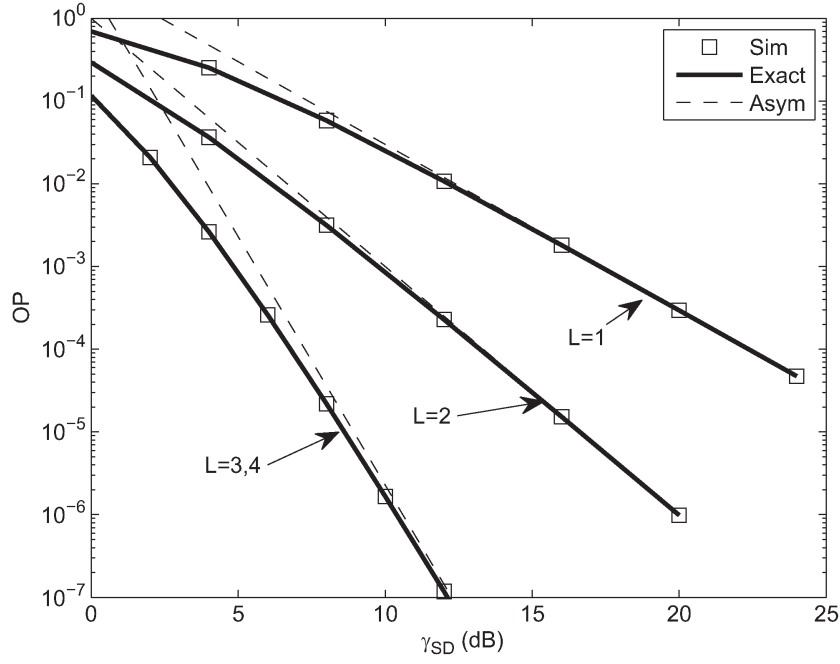

(b)

Fig. 4. OP of MRS as a function of $L$. Setup: $M=3, N=5$ and $\mathcal{R}=M /(M+L)$. (a) $\bar{\gamma}_{S D}=\bar{\gamma}_{S R}=\bar{\gamma}_{R D}$. (b) $\left(\bar{\gamma}_{S D}, \bar{\gamma}_{S R}, \bar{\gamma}_{R D}\right)=(\bar{\gamma}, \bar{\gamma}+9 \mathrm{~dB}, \bar{\gamma}+9 \mathrm{~dB})$.

Fig. 3 shows the OP of SRS-based NCC as a function of $N$. If $N=1$, no RS is applied. It is shown that the proposed mathematical framework closely overlaps with Monte Carlo simulations and that SRS achieves second-order diversity. If the number of available relays $N>1$ increases, SRS provides a better OP in the low-SNR but the same OP in the high-SNR. This is in agreement with Theorem 2, which states that the asymptotic OP of SRS only depends on the source-to-destination links. The gain of RS compared to the baseline setup with $N=1$, i.e., no RS, is equal to $3 \mathrm{~dB}$ in Fig. 3(a) and to $1 \mathrm{~dB}$ in Fig. 3(b).

Fig. 4 shows the OP of MRS-based NCC as a function of $L$. Monte Carlo simulations confirm the accuracy of the proposed mathematical framework and the correctness of the diversity analysis. If $L<M$, in particular, MRS is not capable of achieving full diversity. The diversity order increases with $L$. If $L \geq M$, on the other hand, MRS achieves full diversity and almost the same OP is obtained for all values of $L \geq M$, as a result from Theorem 4.
Fig. 5 compares the OP of MRS-based NCC and repetitionbased NCC as a function of $L$. It confirms the findings of Section VI. In particular, MRS-based NCC outperforms repetition-based NCC if $L \geq M$. If $L<M$, on the other hand, MRS-based NCC outperforms repetition-based NCC in the lowSNR regime but it provides a worse OP in the high-SNR regime because of the lower diversity order $(L+1$ instead of $N+1)$.

Fig. 6 compares the OP of MRS-based NCC and timesharing relaying. It is shown, in particular, that MRS-based NCC outperforms time-sharing relaying if the average SNR of the source-to-destination links is better than that of the relayto-destination links.

\section{CONCLUSION}

In this paper, we have studied outage probability and diversity order of a cooperative protocol that combines relay selection and network coding. A mathematical framework has 


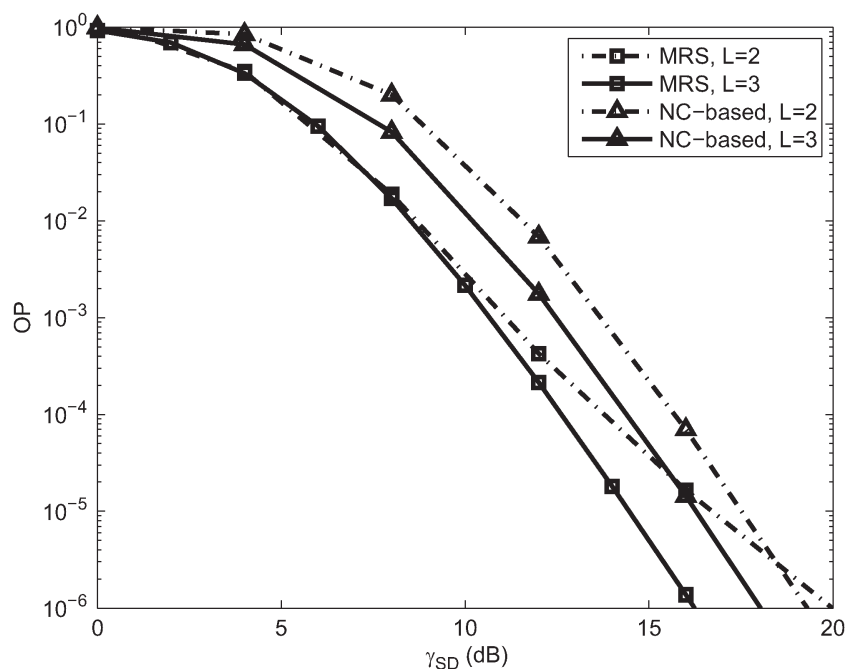

(a)

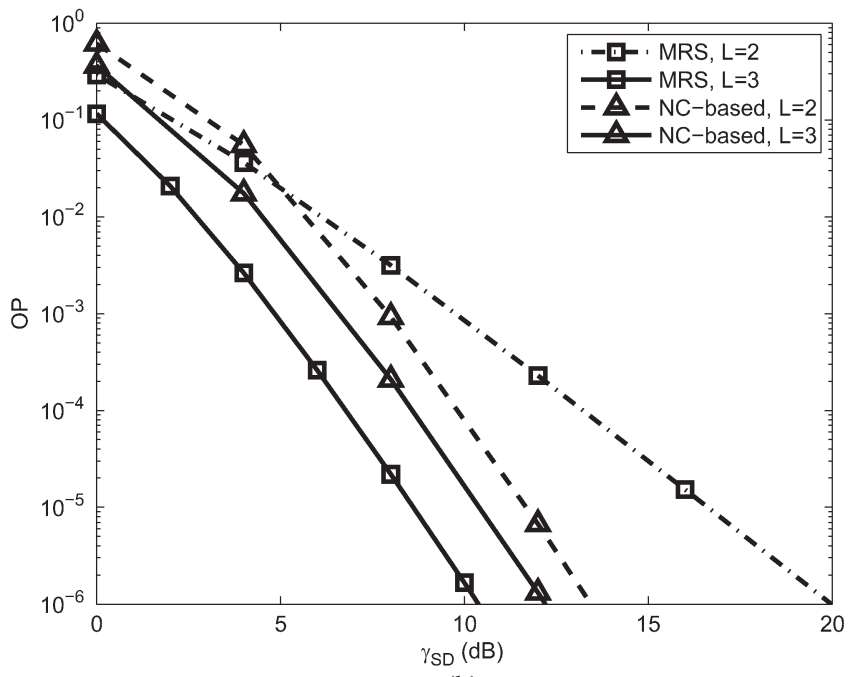

(b)

Fig. 5. OP of MRS-based NCC and repetition-based NCC: Comparison as a function of $L$. Setup: $M=3, N=5, \mathcal{R}=3 / 5$ if $L=2$ and $\mathcal{R}=1 / 2$ if $L=3$. (a) $\bar{\gamma}_{S D}=\bar{\gamma}_{S R}=\bar{\gamma}_{R D}$. (b) $\left(\bar{\gamma}_{S D}, \bar{\gamma}_{S R}, \bar{\gamma}_{R D}\right)=(\bar{\gamma}, \bar{\gamma}+9 \mathrm{~dB}, \bar{\gamma}+9 \mathrm{~dB})$.
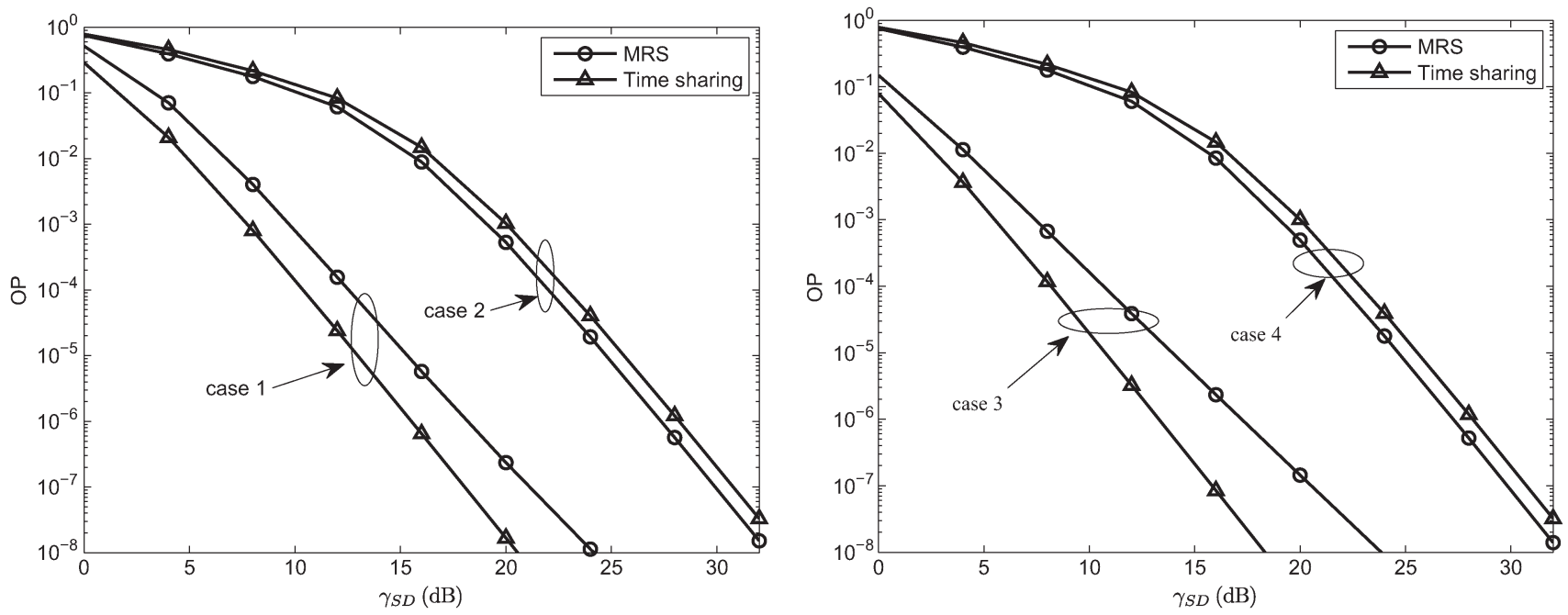

Fig. 6. OP of MRS-based NCC and time-sharing relaying. Setup: $M=3, N=3, L=2$ and $\mathcal{R}=3 / 5$. Case 1: $\bar{\gamma}_{S R}=\bar{\gamma}_{R D}=\bar{\gamma}_{S D}$. Case 2: $\bar{\gamma}_{S R}=\bar{\gamma}_{S D}$, $\bar{\gamma}_{R D}=\bar{\gamma}_{S D}-20 \mathrm{~dB}$. Case 3: $\bar{\gamma}_{S R}=\bar{\gamma}_{S D}+20 \mathrm{~dB}, \bar{\gamma}_{R D}=\bar{\gamma}_{S D}$. Case 4: $\bar{\gamma}_{S R}=\bar{\gamma}_{S D}+20 \mathrm{~dB}, \bar{\gamma}_{R D}=\bar{\gamma}_{S D}-20 \mathrm{~dB}$.

been provided for analyzing the system diversity order. Our analysis has shown that full diversity can be achieved if the number of active relays is at least equal to the number of sources. Otherwise, the diversity order is equal to the number of active relays plus one.

\section{APPENDIX A}

\section{PROOF OF THEOREM 2}

The proof is based on the second-order approximation of $e^{-x}, \forall x>0$, as follows:

$$
e^{-x} \approx 1-x+\frac{x^{2}}{2}+\mathcal{O}\left[x^{3}\right]
$$

Let $a_{m}=\frac{\gamma_{t h}}{\bar{\gamma}_{S_{m} D}}>0, A=\sum_{m=1}^{M} a_{m}$, and $A_{\sim m}=\sum_{m \neq m^{\prime}=1}^{M} a_{m^{\prime}}$. Then, we have:

$$
\prod_{m=1}^{M}\left(1-F_{\gamma_{S_{m} D}}\left(\gamma_{t h}\right)\right)=\prod_{m=1}^{M} e^{-a_{m}}=e^{-A} \approx 1-A+\frac{A^{2}}{2},
$$

and

$$
\begin{aligned}
& \sum_{m=1}^{M} F_{\gamma_{S_{m} D}}\left(\gamma_{t h}\right) \prod_{m^{\prime}=1, m^{\prime} \neq m}^{M}\left(1-F_{\gamma_{S_{j} D}}\left(\gamma_{t h}\right)\right) \\
& \approx \sum_{m=1}^{M}\left(a_{m}-\frac{a_{m}^{2}}{2}\right)\left(1-A_{\sim m}+\frac{A_{\sim m}^{2}}{2}\right) \\
& =\sum_{m=1}^{M} a_{m}-\frac{1}{2} \sum_{\substack{m=1 \\
M}}^{M} a_{m}^{2}-\sum_{m=1}^{M} a_{m} A_{\sim m} \\
& =A-\frac{A^{2}}{2}-\sum_{\substack{m=1, m^{\prime}=1 \\
m \neq m^{\prime}}}^{M} a_{m} a_{m^{\prime}} .
\end{aligned}
$$

Let $b_{n}=\frac{\gamma_{t h}}{\bar{\gamma}_{n}}$ with $1 \leq n \leq N$ and $B=\prod_{n=1}^{N} b_{n}$. Then:

$$
F_{g_{S R S}}\left(\gamma_{t h}\right) \approx \prod_{n=1}^{N}\left(b_{n}-\frac{b_{n}^{2}}{2}\right) \approx B
$$




$$
\left[\begin{array}{llll}
\Psi(0) F_{g_{1}}\left(\gamma_{t h}\right) & & & \\
\Psi(1) F_{g_{1}}\left(\gamma_{t h}\right) & \Psi(0) F_{g_{2}}\left(\gamma_{t h}\right) & & \\
\ldots & & \Psi(0) F_{g_{L}}\left(\gamma_{t h}\right) & \\
\Psi(L-1) F_{g_{1}}\left(\gamma_{t h}\right) & \ldots & \Psi(1) F_{g_{L}}\left(\gamma_{t h}\right) & \Psi(0)\left(1-F_{g_{L}}\left(\gamma_{t h}\right)\right) \\
\Psi(L) F_{g_{1}}\left(\gamma_{t h}\right) & \ldots & \Psi(M-L) F_{g_{L}}\left(\gamma_{t h}\right) & \Psi(M-L-1)\left(1-F_{g_{L}}\left(\gamma_{t h}\right)\right)
\end{array}\right]
$$

$$
\left[\begin{array}{llll}
\Psi(0) F_{g_{1}}\left(\gamma_{t h}\right) & & & \\
\Psi(1) F_{g_{1}}\left(\gamma_{t h}\right) & \Psi(0) F_{g_{2}}\left(\gamma_{t h}\right) & & \\
\ldots & & \ldots & \Psi(0) F_{g_{M}}\left(\gamma_{t h}\right)
\end{array}\right]
$$

The proof follows by inserting (41), (42), and (43) in (17) and by keeping the dominant elements.

\section{APPENDIX B \\ PROOF OF THEOREM 3}

Let divide the packets received at the destination in two groups. The first group consists of the $M$ packets received from the sources. The second group consists of the $L$ packets received from the selected relays. The probability that the destination is capable of decoding $P$ packets is equal to the probability that the SNRs of $P$ links are above the reliability threshold $\gamma_{t h}$. Since these links are uniformly distributed in the two groups, this probability is given by the summation of $\mathcal{C}_{2}^{P}$ terms.

Let $\Psi(k)$ be the probability that there are $k$ source-todestination links whose SNR is above the reliability threshold $\gamma_{t h}$, while the SNRs of the other $M-k$ links are below $\gamma_{t h}$. Since these SNRs are all independent, we obtain (23). The probability that the SNRs of $l<L$ links in $\mathbf{g}_{M R S}$ are above the threshold is given in Lemma 2, which is equal to $F_{g_{l+1}}\left(\gamma_{t h}\right)$. The probability that the SNRs of all $L$ channels in $\mathbf{g}_{M R S}$ are above the threshold is equal to $1-F_{g_{L}}\left(\gamma_{t h}\right)$.

Let consider the probabilities in (20). If $M>L$, the matrix of size $(M+1) \times(L+1)$ shown in (44), shown at the top of the page, can be obtained. If $L \geq M$, the matrix in (45), shown at the top of the page. canbe obtained, instead. We note that the entries in the diagonals have the same $\Psi(k)$. By summing the first $L$ diagonals in (44), we obtain the first factor in (21). By summing the other diagonals in (44), we obtain the second factor in (21). If $L \geq M$, summing the diagonals in (45) and grouping the elements with the same common part yields (22). This concludes the proof.

\section{REFERENCES}

[1] A. Sendonaris, E. Erkip, and B. Aazhang, "User cooperation diversityPart I: System description," IEEE Trans. Commun., vol. 51, no. 11, pp. 1927-1938, Nov. 2003.

[2] A. Bletsas, A. Khisti, D. P. Reed, and A. Lippman, "A simple cooperative diversity method based on network path selection," IEEE J. Sel. Areas Commun., vol. 24, no. 3, pp. 659-672, Mar. 2006.
[3] M. Di Renzo, M. Iezzi, and F. Graziosi, "On diversity order and coding gain of multisource multirelay cooperative wireless networks with binary network coding," IEEE Trans. Veh. Technol., vol. 62, no. 3, pp. 1138-1157, Mar. 2013.

[4] R. Ahlswede, C. Ning, S. Y. R. Li, and R. W. Yeung, "Network information flow," IEEE Trans. Inf. Theory, vol. 46, no. 4, pp. 1204-1216, Apr. 2000.

[5] Z. Ding and K. Leung, "On the combination of cooperative diversity and network coding for wireless uplink transmissions," IEEE Trans. Veh. Technol., vol. 60, no. 4, pp. 1590-1601, Apr. 2011.

[6] Y. Li, R. H. Y. Louie, and B. Vucetic, "Relay selection with network coding in two-way relay channels," IEEE Trans. Veh. Technol., vol. 59, no. 9, pp. 4489-4499, Sep. 2010.

[7] Y. Jing and H. Jafarkhani, "Single and multiple relay selection schemes and their achievable diversity orders," IEEE Trans. Wireless Commun., vol. 8, no. 3, pp. 1414-1423, Mar. 2009.

[8] S. Atapattu, Y. Jing, H. Jiang, and C. Tellambura, "Relay selection schemes and performance analysis approximations for two-way networks," IEEE Trans. Commun., vol. 61, no. 3, pp. 987-998, Mar. 2013.

[9] M. Di Renzo, M. Iezzi, and F. Graziosi, "Error performance and diversity analysis of multi-source multi-relay wireless networks with binary network coding and cooperative MRC," IEEE Trans. Wireless Commun., vol. 12, no. 6, pp. 2883-2903, Jun. 2013.

[10] M. Di Renzo, "On the achievable diversity of repetition-based and relay selection network-coded cooperation," IEEE Trans. Commun., vol. 62, no. 7, pp. 2296-2313, Jul. 2014.

[11] C. Peng, Q. Zhang, M. Zhao, Y. Yao, and W. Jia, "On the performance analysis of network-coded cooperation in wireless networks," IEEE Trans. Wireless Commun., vol. 7, no. 8, pp. 3090-3097, Aug. 2008.

[12] H. Topakkaya and W. Zhengdao, "Wireless network code design and performance analysis using diversity-multiplexing tradeoff," IEEE Trans. Commun., vol. 59, no. 2, pp. 488-496, Feb. 2011.

[13] Z. Ding, T. Ratnarajah, and K. Leung, "On the study of network coded AF transmission protocol for wireless multiple access channels," IEEE Trans. Wireless Commun., vol. 7, no. 11, pp. 4568-4574, Nov. 2008.

[14] W. Geng and T. Lv, "An opportunistic network coding cooperative scheme for wireless uplink multi-relay cooperative networks," in Proc. Int. Symp. Wireless Pers. Multimedia Commun., Sep. 2012, pp. 148-152.

[15] W. Guan and K. Liu, "Diversity analysis of analog network coding with multi-user interferences," IEEE Trans. Wireless Commun., vol. 12, no. 2 , pp. 668-679, Feb. 2013.

[16] M. K. Simon and M.-S. Alouini, Digital communication over fading channels, 2nd ed. London, U.K.: Wiley, 2005.

[17] R. C. Singleton, "Maximum distance Q-Nary codes," IEEE Trans. Inf. Theory, vol. 10, no. 2, pp. 116-118, Feb. 1964.

[18] A. Behnad, A. Rabiei, N. Beaulieu, and H. Hajizadeh, "Generalized analysis of dual-hop DF opportunistic relaying with randomly distributed relays," IEEE Commun. Lett., vol. 17, no. 6, pp. 1057-1060, Jun. 2013. 


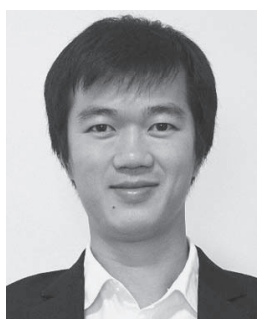

Thang X. Vu (S'11-M'15) was born in Hai Duong, Vietnam, in 1985. He received the B.S. and M.Sc. degrees in electronics and telecommunications engineering from the VNU University of Engineering and Technology, Vietnam, in 2007 and 2009, respectively, and the Ph.D. degree in electrical engineering from the University Paris-Sud, France, in 2014.

From 2007 to 2009, he was a Research Assistant at the Department of Electronics and Telecommunications, VNU University of Engineering and Technology, Vietnam. In 2010, he received the Allocation de Recherche fellowship to study Ph.D. in France. From September 2010 to May 2014, he was with the Laboratory of Signals and Systems (LSS), a joint laboratory of CNRS, CentraleSupelec and University Paris-Sud XI, France. Since July 2014, he has been a Postdoctoral Research Fellow at the Information Systems Technology and Design (ISTD) Pillar, Singapore University of Technology and Design (SUTD), Singapore. His research interests are in the field of wireless communications, with particular interests of cloud radio access networks (C-RAN), cooperative diversity, channel coding, network decoding, and iterative decoding.

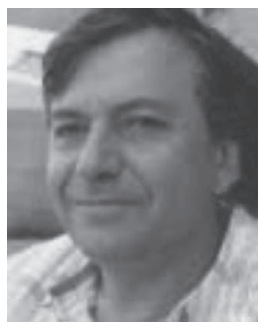

Pierre Duhamel (F'98) was born in France in 1953. $\mathrm{He}$ received the B.Eng. degree in electrical engineering from the National Institute for Applied Sciences (INSA) Rennes, France, in 1975, the Dr.Eng. degree in 1978, and the Doctorat ès sciences degree in 1986, both from Orsay University, Orsay, France.

From 1975 to 1980 , he was with Thomson-CSF (now Thales), Paris, France, where his research interests were in circuit theory and signal processing, including digital filtering and analog fault diagnosis. In 1980, he joined the National Research Center in Telecommunications (CNET, now Orange Labs), Issy les Moulineaux, France, where his research activities were first concerned with the design of recursive CCD filters. Later, he worked on fast algorithms for computing Fourier transforms and convolutions, and applied similar techniques to adaptive filtering, spectral analysis and wavelet transforms. From 1993 to September 2000, he was a Professor at ENST, Paris (National School of Engineering in Telecommunications) with research activities focused on Signal processing for Communications. He was head of the Signal and Image Processing Department from 1997 to 2000. He is now with CNRS/L2S (Laboratoire de Signaux et Systemes, Gif sur Yvette, France), where he is developing studies in signal processing for communications (including equalization, iterative decoding, multi-carrier systems, cooperation) and signal/image processing for multimedia applications, including joint source-protocol-channel coding. He is currently investigating the connections between communication theory and networking. He has published more than 93 papers in international journals, more than 300 papers in international conferences, and holds 29 patents. He is a co-author of the book Joint Source and Channel Decoding: A Cross Layer Perspective With Applications In Video Broadcasting (Academic Press, 2009).

He was chairman of the DSP committee from 1996 to 1998, a member of the SP for Com committee until 2001, and a member of the IEEE Signal Processing for Communications and Networking Technical Committee from 2004 to 2010. He was an Associate Editor of the IEEE TRANSACTIONS ON Signal PRocessing from 1989 to 1991, an Associate Editor for the IEEE Signal Processing LetTers, and a Guest Editor for the special issue of the IEEE Transactions On Signal Processing on wavelets. $\mathrm{He}$ was Distinguished Lecturer, IEEE, for 1999, and was co-technical chair of ICASSP 06, Toulouse, France, and of WCNC 2012, Paris, France. The paper on subspace-based methods for blind equalization, which he co-authored, received the Best Paper Award from the IEEE Transactions on Signal PROCESSING in 1998. He was awarded the Grand Prix France Telecom by the French Science Academy in 2000. He is a Fellow, EURASIP, since 2008.

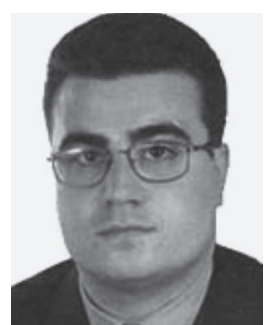

Marco Di Renzo (S'05-AM'07-M'09-SM' 14) was born in L'Aquila, Italy, in 1978. He received the Laurea (cum laude) and the Ph.D. degrees in electrical and information engineering from the Department of Electrical and Information Engineering, University of L'Aquila, Italy, in April 2003 and January 2007, respectively. In October 2013, he received the Habilitation à Diriger des Recherches (HDR) from University Paris-Sud XI, Paris, France.

Since January 2010, he has been a CNRS Associate Research Professor ("Chargé de Recherche Titulaire CNRS") in the Laboratory of Signals and Systems (UMR-8506) of Paris-Saclay University, a joint academic and research laboratory of CNRS, CentraleSupelec and University Paris-Sud XI, Paris, France. His main research interests are in the field of wireless communications theory.

From August 2002 to January 2008, he was with the Center of Excellence for Research DEWS, University of L'Aquila, Italy. In the fall of 2006, he was a Visiting Scholar in the Bradley Department of Electrical and Computer Engineering, Virginia Tech, Blacksburg, VA, USA. From February 2008 to April 2009, he was a tenured Research Associate with the Telecommunications Technological Center of Catalonia (CTTC), Spain. From May 2009 to December 2009, he was an EPSRC Research Fellow with the Institute for Digital Communications (IDCOM), The University of Edinburgh, U.K.

Dr. Di Renzo is the recipient of a special mention for the outstanding five-year (1997-2003) academic career, University of L'Aquila, Italy; the THALES Communications Ph.D. fellowship (2003-2006), University of L'Aquila, Italy; the 2004 Best Spin-Off Company Award, Abruzzo Province, Italy; the 2008 Torres Quevedo Award, Ministry of Science and Innovation, Spain; the "Dérogation pour l'Encadrement de Thèse" (2010), University ParisSud XI, France; the 2012 IEEE CAMAD Best Paper Award; the 2012 IEEE Wireless COMMUniCATIONS LeTters Exemplary Reviewer Certificate; the 2013 IEEE VTC-Fall Best Student Paper Award; the 2013 Network of Excellence NEWCOM $\backslash$ \# Best Paper Award; the 2013 IEEE TRANSACTIONS ON Vehicular Technology Top Reviewer Award; the 2013 IEEE-COMSOC Best Young Researcher Award for Europe, Middle East and Africa (EMEA Region); the 2014 Royal Academy of Engineering Distinguished Visiting Fellowship, United Kingdom; the 2014 IEEE ATC Best Paper Award; the 2014 IEEE CAMAD Best Demo Award; the 2014 IEEE CAMAD Best Paper Award; and the 2014 IEEE WIRELESS COMMUNICATIONS LETTERS Exemplary Reviewer Certificate. Currently, he serves as an Editor of the IEEE COMMUNICATIONS LETTERS and of the IEEE TRANSACTIONS ON COMMUNICATIONS (Heterogeneous Networks Modeling and Analysis). 